\title{
Types of Instantaneous Functional Interference Effects on the Autonomic- Cardiovascular Response to the Simultaneous Performance of Cold Face and Active Orthostatic Tests
}

\author{
Salvador Carrasco-Sosa ${ }^{1}$, Alejandra Guillén-Mandujano ${ }^{1}$, Aldo R. Mejía-Rodríguez ${ }^{2}$ \\ ${ }^{1}$ Ciencias Biológicas y de la Salud, Universidad Autónoma Metropolitana-I, CDMX, México \\ ${ }^{2}$ Universidad Autónoma de San Luis Potosí, San Luis Potosí, México
}

\begin{abstract}
In 25 healthy subjects we assessed the effects of cold face test (CFT), active orthostatic test (AOT) and their simultaneous performance (CFAOT) on: RR intervals $(R R)$, systolic pressure (SP), and peak arterial pressure derivative (dmAP) time series, their instantaneous lowand high-frequency powers, baroreflex sensitivity (BRS) and respiratory sinus arrhythmia sensitivity (RSAS), obtained by a time-frequency distribution. The functional interference effect index (FIEI) of each variable was computed as the CFAOT to CFT+AOT ratio. In relation to CFT+AOT, CFAOT showed: greater initial sympathetic estimators overshoots, associated to greater $S P$ and dmAP increments and RR, BRS and RSAS undershoots, all with FIEI>1; followed by vagal increase and gradual recoveries of BRS, RSAS, sympathetic and cardiovascular estimators with FIEI $>1$. At the end, all variables but $R R$ presented FIEI $<1$. CFAOT initially induces a potentiative sympathetic activation associated to great reductions of $R R, B R S$ and $R S A S$, and potentiative SP and dmAP increases, followed by potentiative sympathetic recovery and vagal activation with potentiative RR, BRS, RSAS, SP and dmAP gradual recoveries that become suppressive at the end. FIEI of all variables fluctuates between suppressive and potentiative, showing the convergence of autonomic inputs.
\end{abstract}

\section{Introduction}

It is commonly accepted that active orthostatic test (AOT) provokes an increase in sympathetic activity with baroreflex involvement $[1,2]$ and that cold face test (CFT) elicits a non-baroreflex sympatho-vagal coactivation $[2$, $3]$. However, when we recently compared the time course of the autonomic cardiovascular effects of CFT and AOT, we found that their response patterns are quite opposite, because, while CFT elicits great increases in vagal activity, baroreflex sensitivity (BRS) and respiratory sinus arrhythmia sensitivity (RSAS), AOT shows a 7 times greater sympathetic activation, and BRS and RSAS reductions [4,5]. If CFT and AOT were performed simultaneously (CFAOT), how would the minimal sympathetic activity of the former interfere with the sympathetic predominance of the latter? And how would the minimal vagal outflow of AOT interfere with the great vagal activation of CFT? To answer these questions, we modified an experimental strategy that has been employed to evaluate the suppressive and facilitatory interactions in the electrical response of sensory cortical areas $[6,7]$. Recently, we studied the interference effects between regions of the autonomic nervous system when stimulated by complex respiratory maneuvers [8].

Our methodological strategy was to compare the responses to two different maneuvers applied separately and simultaneously, testing the assumption that, if the response to the simultaneous maneuver equals the sum of the separate responses, no functional interference effects (FIE) are produced, so, there is no convergence of inputs. Thus, we assessed the time course of FIE provoked by CFAOT relative to the sum of the separate effects of CFT and AOT (CFT+AOT) on the instantaneous spectral measures derived from heart rate and arterial pressure (AP) variabilities, BRS and RSAS.

\section{Methods}

\subsection{Subjects}

Twenty-five healthy, normotensive and sedentary subjects, 14 men and 11 women, were studied. Mean age, height and weight were $22.2 \pm 2.2$ years, $167 \pm 8 \mathrm{~cm}$ and $69.1 \pm 10.4 \mathrm{~kg}$ respectively. Their written informed consent was requested to participate.

\subsection{Protocol}

Volunteers visited the laboratory twice, first to evaluate their health status and anthropometric values, and second to carry out the experimental stage. Subjects 
underwent 1-min control, 1-min maneuver and 2-min recovery stages for CFT, AOT and CFAOT, applied in random order. To perform AOT, the subjects rapidly stood up from the supine position, returning to this position at the end of the maneuver stage. CFT was done by applying a bag filled with ice water at $0^{\circ} \mathrm{C}$ on the face, excluding the eyes, with the subject in supine position. The bag was removed at the end. For CFAOT, the ice bag was placed first and then subjects stood up. At the end, subject first lied down and then the bag was removed.

\subsection{Signal recording and acquisition}

ECG was detected at the CM5 bipolar lead using a bioelectric amplifier (Biopac Systems). AP was measured by Finapres (Ohmeda). Respirogram (Res) was obtained by Inductotrace (Ambulatory Monitoring). All signals were digitized at a sampling rate of $1 \mathrm{kHz}$ via an acquisition system (Biopac Systems).

\subsection{Data processing}

R-wave peaks, systolic pressure (SP), diastolic pressure (DP), maximal amplitudes of AP derivative (dmAP) and Res were detected to construct the time series of R-R intervals (RR), SP, DP, their difference, pulse pressure (PP), dmAP and Res. These series were cubic-spline interpolated, resampled at $4 \mathrm{~Hz}$ and detrended. Time-frequency spectra of the series were estimated with the smoothed pseudo-Wigner-Ville distribution and integrated in the standard low frequency (LF) and high frequency (HF) bands to compute the instantaneous values of LF powers of RR $\left(\mathrm{LF}_{\mathrm{RR}}\right)$, SP $\left(\mathrm{LF}_{\mathrm{SP}}\right), \mathrm{PP}\left(\mathrm{LF}_{\mathrm{PP}}\right)$ and dmAP $\left(\mathrm{LF}_{\mathrm{dmAP}}\right)$ and HF powers of $\mathrm{RR}\left(\mathrm{HF}_{\mathrm{RR}}\right)$ and Res $\left(\mathrm{HF}_{\mathrm{Res}}\right)$. Instantaneous $\mathrm{BRS}$ and RSAS were computed by alpha index. The required timefrequency coherences were considered significant when greater than 0.5. The instantaneous difference of CFAOT and CFT+AOT and the FIE index (FIEI) as their ratio were computed for each variable. After subtracting their mean baseline value, the individual dynamics of all the variables were ensemble-averaged for visualization, and segmented into 6-s epochs for statistical analysis.

\subsection{Statistical analysis}

Data are expressed as mean \pm SD. CFAOT vs. CFT+AOT and FIEI vs. 1 differences were tested by paired t-test with a significance level of $\mathrm{p}<0.05$.

\section{Results}

During AOT, time-frequency spectra of RR series showed two overshoots in $\mathrm{LF}_{\mathrm{RR}}$ and minimal fluctuations in $\mathrm{HF}_{\mathrm{RR}}$ (Fig. 1A). In CFT, the opposite response was observed: reduction of $\mathrm{LF}_{\mathrm{RR}}$ and large increase of $\mathrm{HF}_{\mathrm{RR}}$ (Fig. 1B). Meanwhile, in response to CFAOT, $\mathrm{LF}_{\mathrm{RR}}$ power showed only an initial overshoot and a final $\mathrm{HF}_{\mathrm{RR}}$ overshoot, both of greater amplitude than those of AOT and CFT, respectively (Fig. 1C).

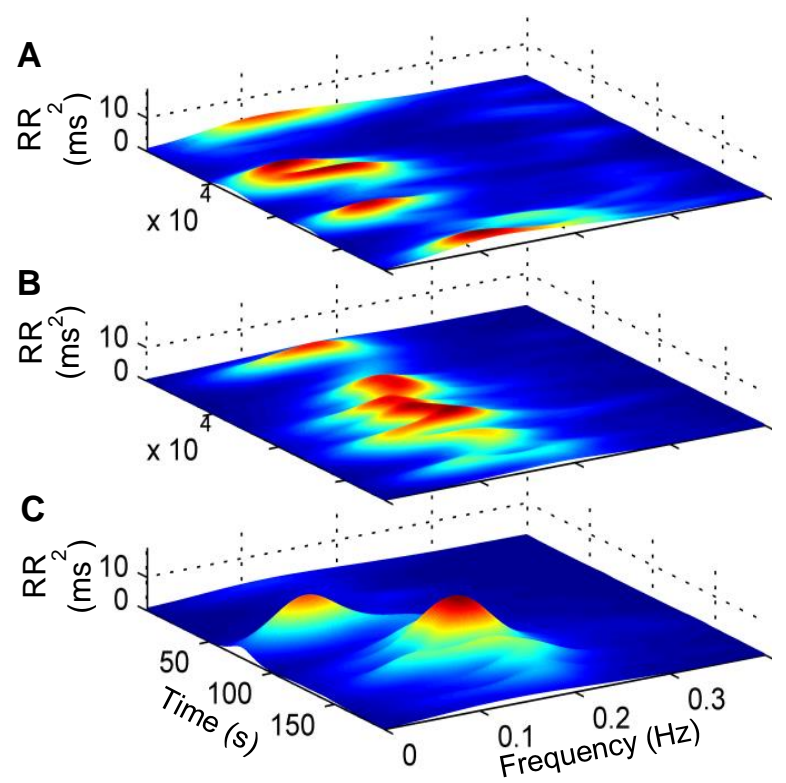

Fig. 1. Representative time-frequency distributions of RR during A) AOT, B) CFT and C) CFAOT. Maneuver spans from 60 to $120 \mathrm{~s}$.

All of the ensemble averages of the studied variables showed consistent response patterns in the three maneuvers. The 6-s epochs pooled mean values of CFAOT response vs. those of CFT+AOT were: during the initial $18 \mathrm{~s}$, greater in $\mathrm{LF}_{\mathrm{PP}}(\mathrm{p}<0.007$; Fig. $2 \mathrm{~A})$ and $\mathrm{LF}_{\mathrm{dmAP}}$ ( $p<0.001$; Fig. 2B) overshoots but smaller in the $\mathrm{LF}_{\mathrm{SP}}$ one ( $<<0.01$; Fig. 2C) and similar for $\mathrm{HF}_{\mathrm{RR}}$ power (Fig. $2 \mathrm{H}$ ); after a brief undershoot, greater peak mean values were reached by dmAP $(p<0.001$; Fig. $2 E)$ and $S P(p<0.001$; Fig. 2F); RR ( $p<0.001$; Fig. 2G), BRS ( $<<0.04$; Fig. $2 \mathrm{H}$ ) and RSAS $(p<0.01$; Fig. 2I) presented deeper undershoots. From $18 \mathrm{~s}$ to the beginning of recovery at 62 $\mathrm{s}, \mathrm{HF}_{\mathrm{RR}}$ progressively increased with greater mean values ( $<<0.04$; Fig. $2 \mathrm{H}$ ) that became similar at the ending; mean values of both the maximal increase and the gradual recovery of RR were greater $(\mathrm{p}<0.001$; Fig. $2 \mathrm{G})$, as well as the means of the progressive return to baseline of $\mathrm{LF}_{\mathrm{PP}}$ $\left(p<0.01 ;\right.$ Fig. 2A), LF $_{d m A P}(p<0.01 ;$ Fig. $2 B), L_{R R}$ $(\mathrm{p}<0.02$; Fig. 2D), dmAP $(\mathrm{p}<0.02$; Fig. 2E) and $S P$ ( $p<0.001$; Fig. 2F). Mean values of $\mathrm{LF}_{\mathrm{SP}}$ (Fig. 2C) were similar during their gradual recovery but became smaller $(\mathrm{p}<0.01)$ at the end. The increase of RSAS ( $<<0.04$; Fig. $2 \mathrm{~J})$ and the progressive recovery of BRS ( $<<0.02$; Fig. $2 \mathrm{I}$ ) had smaller means. With the exception of RR, whose mean values remained greater $(p<0.002$; Fig. $2 G)$ than CFT+AOT upon the end, mean values of the rest of the variables became smaller ( $<<0.001$; Fig. 2). 


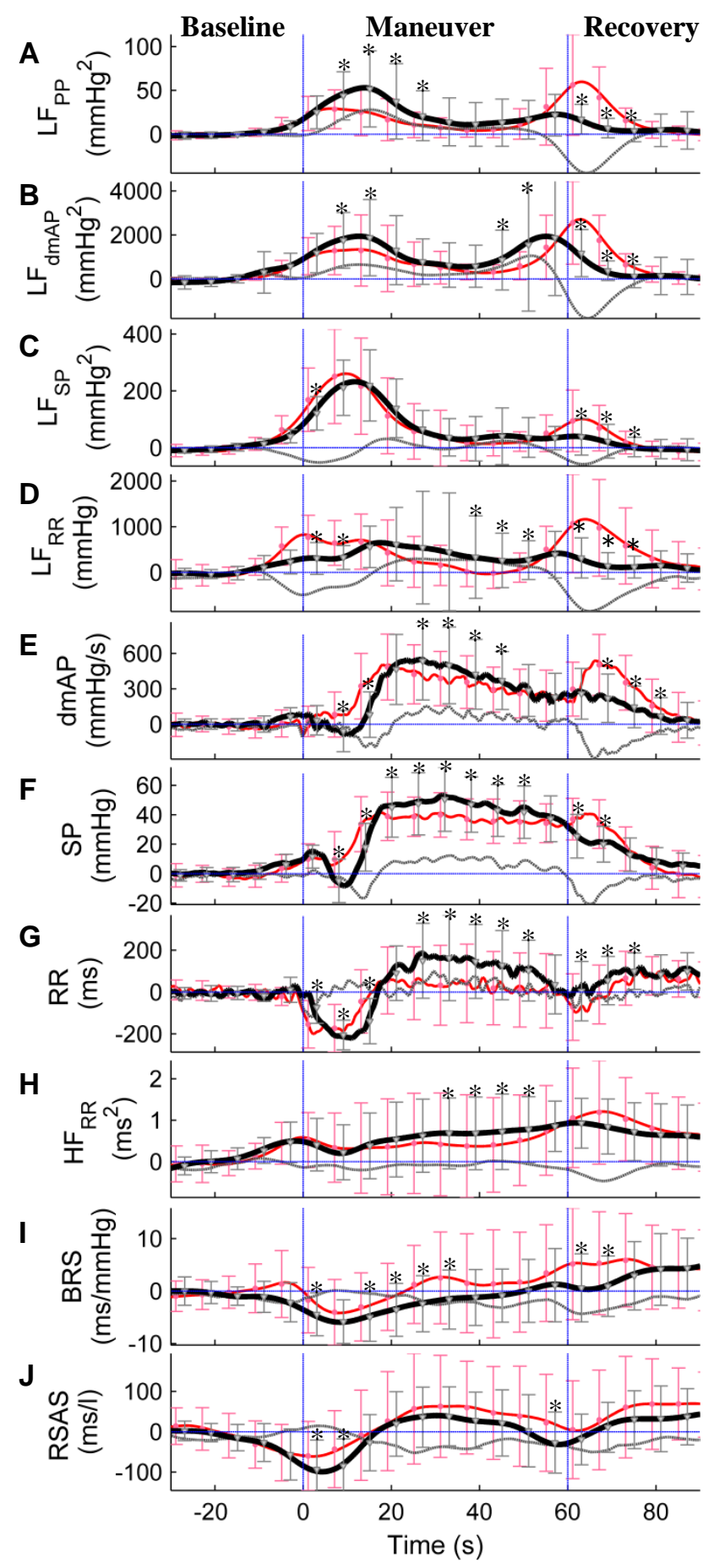

Fig. 2. Ensemble averages and 6-s epoch means $\pm \mathrm{SD}$ of: A) $\left.\mathrm{LF}_{\mathrm{PP}}, \mathrm{B}\right) \mathrm{LF}_{\mathrm{dmAP}}$, C) $\mathrm{LF}_{\mathrm{SP}}$, D) $\mathrm{LF}_{\mathrm{RR}}$, E) dmAP, F) $\mathrm{SP}$, G) RR, H) $\mathrm{HF}_{\mathrm{RR}}$, I) BRS and J) RSAS in CFAOT (thick black line), CFT+AOT (thin red line) and their difference (dotted grey line) . $* \mathrm{p}<0.04$ CFAOT

In relation to level=1, time course of the FIEI showed fluctuations in type and amplitude, both intra- and interestimators (Fig. 3). For instance, RR presented the greatest brief initial FIEI $>1 \quad(p<0.001)$, followed by
FIEI $<1(p<0.001)$ and then a sustained FIEI $>1(p<0.001)$ that increases even more at the end $(\mathrm{p}<0.01) ; \mathrm{HF}_{\mathrm{RR}}$ initially presented FIEI $=1$ followed by FIEI $>1 \quad(p<0.02)$ and a final FIEI=1; in SP response, after the initial FIEI $<1$ $(p<0.001)$ there was a slight sustained FIEI $>1 \quad(p<0.001)$ that also became FIEI $<1 \quad(\mathrm{p}<0.001)$ at the end; and with the smallest FIE, $\mathrm{LF}_{\mathrm{SP}}$ showed in most of the maneuver a FIEI $=1$, that turned into FIEI $<1(p<0.02)$ in the end.

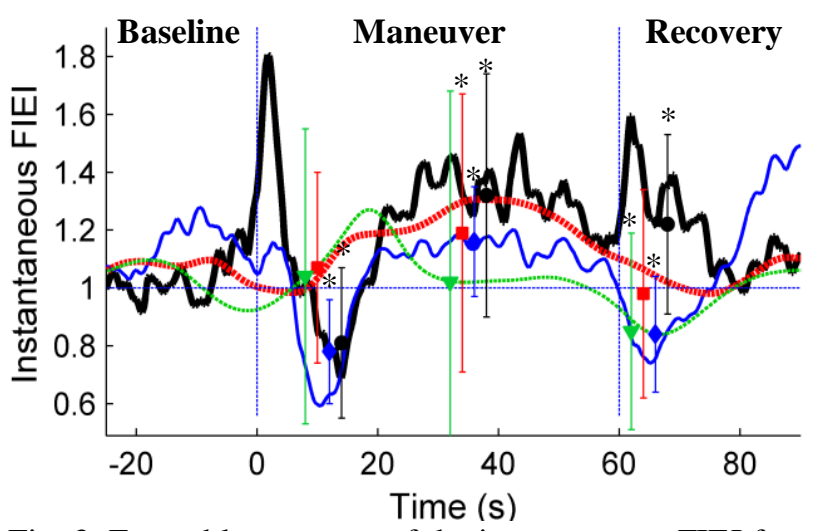

Fig. 3. Ensemble averages of the instantaneous FIEI for $\mathrm{RR}$ (thick black solid line), $\mathrm{HF}_{\mathrm{RR}}$ (thick red dashed line), SP (thin blue solid line) and $\mathrm{LF}_{\mathrm{SP}}$ (thin green dashed line). For clarity, only mean values at 14, 38 and $68 \mathrm{~s}$ are shown. ${ }^{*} \mathrm{p}<0.04$ FIEI vs. 1.

\section{Discussion}

Our study documents the interactions between convergent inputs of the autonomic nervous system in humans, by showing that their FIEI fluctuates between suppressive and potentiative throughout the time course of each variable and with varying amplitudes among the variables: relative to $\mathrm{CFT}+\mathrm{AOT}$, CFAOT response presents initial overshoots of greater magnitude in $\mathrm{LF}_{\mathrm{PP}}$ and $\mathrm{LF}_{\mathrm{dmAP}}$, similar in $\mathrm{HF}_{\mathrm{RR}}$ and smaller in $\mathrm{LF}_{\mathrm{SP}}$; larger abrupt rises of SP and dmAP; and deeper undershoots, followed by abrupt increases in RR and RSAS; followed until the end by greater values in the progressive increase of $\mathrm{HF}_{\mathrm{RR}}$, and the progressive recovery of $\mathrm{LF}_{\mathrm{PP}}, \mathrm{LF}_{\mathrm{dmAP}}$, $\mathrm{LF}_{\mathrm{RR}}, \mathrm{RR}, \mathrm{dmAP}$ and $\mathrm{SP}$; lesser values in the progressive recovery of BRS and RSAS and similar in the $\mathrm{LF}_{\mathrm{SP}}$ recovery. Upon the termination, all estimators are smaller, except RR.

To the best of our knowledge, this is the first study to establish the time course and the type of FIE in the autonomic-cardiovascular effects provoked by CFAOT, which begin as sympathetic incremental potentiative FIE, followed by incremental sympathetic and vagal potentiative FIE that become suppressive after the end.

The method for assessing neural networks interactions has been used to evaluate the degree of interaction and therefore, convergence, of different sensory inputs in human somatosensory cortex [6,7]. Recently, we 
translated this methodology to the study of autonomic cardiovascular control to evaluate the FIE provoked by fluctuating respiratory patterns on $\mathrm{HF}_{\mathrm{RR}}$ power [8]. In the present study, our methodology is further improved by: considering complex responses, as those of AOT and CFT; tracking the time-course of a battery of estimators including our proposed sympathetic activity indexes, $\mathrm{LF}_{\mathrm{PP}}$ and $\mathrm{LF}_{\mathrm{dmAP}}[4]$, and, particularly, assessing the FIEI in an instantaneous fashion.

Both the difference between CFAOT and CFT+AOT and the FIEI assess the type and degree of FIE in each variable, but the FIEI also allows comparing among variables. Thus, FIE is: potentiative when the simultaneous effect is greater than the sum of separate effects (difference $>0$ and FIEI $>1$ ), with incremental and decremental cases; or suppressive, when the simultaneous effect is less than the sum of separate effects (difference $<0$ and FIEI $<1)$. When the simultaneous effect is equal to the sum of separate effects (difference $=0$ and FIEI $=1$ ), there is no FIE, that is, there is no input convergence.

Our findings suggest that the physiological mechanisms that generate CFAOT response patterns begin when the activation of central command for standing up and the consequential fall of AP (Fig. 2F) trigger, via baroreflex unloading, a great overshoot of sympathetic activity, whose estimators show, first, some suppressive FIE and later different degrees of incremental potentiative FIE (Fig. 2A-D) that provoke: 1) undershoots in RR, BRS and RSAS, also with potentiative FIE (Fig. $2 \mathrm{G}, \mathrm{I}-\mathrm{J})$, and 2) abrupt increases in contractility and SP with incremental potentiative FIE (Fig. 2E-F). The slight initial overshoot of vagal activity was additive (Fig. 2H). The SP increase, via baroreflex loading (with diminished BRS and decremental potentiative FIE), produces the gradual recovery of sympathetic activity (Fig. 2F, A-D) and the gradual increase of vagal outflow (Fig. $2 \mathrm{H}$ ), both with different degrees of potentiative FIE; the progressive recovery of BRS with decremental potentiative FIE is a contributing factor (Fig. 2I). The gradual vagal increase with potentiative FIE, probably reinforced by the augmented trigeminal activity, provokes abrupt rises of RR and RSAS that then fall steadily, the former with potentiative FIE and the latter with suppressive FIE (Fig. $2 \mathrm{G}, \mathrm{J})$. The progressive return to baseline of sympathetic activity with potentiative FIE, induces the gradual recovery of contractility and $\mathrm{SP}$, both with potentiative FIE (Fig. 2E-F), and contributes to RR shortening. In the termination, with the exception of RR that presents potentiative FIE, all the variables show suppressive FIE (Fig. 2). While the time course of the response to AOT shows two overshoots in the sympathetic estimators and undershoots in $\mathrm{HF}_{\mathrm{RR}}$, RR, BRS and RSAS, in CFT there are overshoots in $\mathrm{HF}_{\mathrm{RR}}$, RR, BRS and RSAS [4, 5]. From the above findings, CFAOT shows potentiation in the sympathetic and vagal indexes, in SP, RR (first decremental, then incremental) and predominance of
AOT- related BRS and RSAS reductions.

In conclusion, our novel methodology for obtaining the time course of the spectral estimators of autonomic cardiovascular control and their FIE in response to CFAOT revealed a highly dynamic functional picture of both the response patterns and the FIEI of all the variables, consisting in an initial sympathetic overshoot with incremental potentiative FIE, associated, on the one hand, to RR, BRS and RSAS undershoots with decremental potentiative FIE and, on the other, to abrupt increases of contractility and AP, whose estimators show incremental potentiative FIE. The return to baseline of the sympathetic and cardiovascular variables, as well as the progressive increase of vagal activity, all involving baroreflex and trigeminal activation, present incremental potentiative FIE. After CFAOT is over, all sympathovagal activity and cardiovascular measures, except RR, present suppressive FIE. Thus, throughout the course of CFAOT all variables present different degrees of suppressive and potentiative FIE.

\section{References}

[1] Jacob G, Ertl AC, Shannon JR, Furlan R, et al. Effect of standing on neurohumoral responses and plasma volume in healthy subjects. J Appl Physiol 1998; 84:914-21.

[2] Hilz MJ, Dütsch M. Quantitative studies of autonomic function. Muscle Nerve 2006; 33:6-20.

[3] Khurana R. Cold face test: adrenergic phase. Clin Auton Res 2007; 17:211-6.

[4] Carrasco-Sosa S, Guillén-Mandujano A, Mejía-Rodríguez A. Beat-to-beat Response Patterns of Spectral Sympathetic Estimators to the Cold Face Test and their Comparison to those of the Active Orthostatic Test. Comput Cardiol 2015;42:21-4.

[5] Carrasco-Sosa S, Guillén-Mandujano A, Mejía-Rodríguez A. Instantaneous Response Patterns of Baroreflex Sensitivity, Respiratory Sinus Arrhythmia Sensitivity and Vagal Activity to Cold Face Test and Active Orthostatic Test. Comput Cardiol, DOI:10.22489/CinC.2016.152-111.

[6] Gandevia S, Burke D, McKeon B. Convergence in the somatosensory pathway between cutaneous afferents from the index and middle fingers in man. Exp Brain Res 1983; 50:415-25.

[7] Cardini F, Longo M, Haggard P. Vision of the body modulates somatosensory intracortical inhibition. Cereb Cortex 2011; 21:2014-22.

[8] Guillén-Mandujano A, Carrasco-Sosa S. Additive effect of simultaneously varying respiratory frequency and tidal volume on respiratory sinus arrhythmia. Auton Neurosci 2014; 186:69-76.

Address for correspondence.

Salvador Carrasco-Sosa

Depto. Ciencias de la Salud, S-353

Universidad Autónoma Metropolitana-Iztapalapa.

Av. San Rafael Atlixco \# 186, C.P. 09340 CDMX, México. scas@xanum.uam.mx 